\section{MALABSORCIÓN DE LACTOSA EN ADOLESCENTES DE UN CLUB DE FÚTBOL}

\author{
MALABSORPTION OF LACTOSE IN \\ ADOLESCENT MEMBERS OF A FOOTBALL CLUB
}

\author{
Roberto A. Accinelli ${ }^{1,2, a}$, Sonia Herrera ${ }^{1, b}$, Yuri \\ Canales $^{1, b}$, Lidia M. López ${ }^{1, b}$
}

Sr Editor. La malabsorción de lactosa (MAL) se produce por ausencia o disminución de la actividad de la enzima lactasa ${ }^{(1)}$. La actividad de la lactasa es máxima durante la infancia y en el adulto permanece elevada solo en aquellos con fenotipo lactasa persistente. La lactosa no absorbida es fermentada por la microflora bacteriana del colón, produciendo ácidos grasos de cadena corta y gases como hidrógeno, metano y dióxido de carbono ${ }^{(2)}$. Estas sustancias provocan diferentes síntomas como dolor abdominal, diarrea, flatos, distensión abdominal, eructos y meteorismo.

La intolerancia a la lactosa (IL) es la aparición de estos síntomas luego de ingerir $12 \mathrm{~g}$ de lactosa, cantidad presente en $240 \mathrm{~mL}$ de leche. El umbral de tolerancia/intolerancia depende no solo de la actividad de la enzima lactasa sino, además de la dosis de lactosa, el vaciamiento gástrico, el tiempo de tránsito intestinal y el tipo y cantidad de flora bacteriana intestinal ${ }^{(1)}$.

Realizamos un estudio para determinar la frecuencia de MAL en un grupo de adolescentes jugadores de fútbol. Para ello, se tomó una muestra no probabilística de 98 futbolistas amateurs del Club Cantolao (Lima-Perú), todos de sexo masculino, a quienes se le realizó la prueba de tolerancia a la lactosa, que tiene una especificidad de 77$96 \%$ y sensibilidad de $76-94 \%{ }^{(3)}$. La prueba consistió en tomar muestras de sangre venosa en cuatro ocasiones, primero se midió la glicemia basal, luego se dio a cada participante una dosis estándar de lactosa $(2 \mathrm{~g} / \mathrm{kg}$ hasta un máximo de $50 \mathrm{~g}$ ) y posterior a la ingesta de esta se tomaron glicemias a los 30,60 y 120 min ${ }^{(4)}$. Se consideró un aumento de glucosa en sangre menor o igual a $20 \mathrm{~g} / \mathrm{dL}$ para el diagnóstico de MAL.

La prueba de tolerancia a la lactosa fue positiva en 54 participantes, con lo que se obtuvo una frecuencia de MAL

\footnotetext{
1 Instituto de Investigaciones de la Altura, Universidad Peruana Cayetano Heredia. Lima, Perú.

2 Servicio de Neumología, Hospital Cayetano Heredia. Lima, Perú.

a Médico neumólogo, magíster en salud pública; ${ }^{\mathrm{b}}$ médico cirujano Recibido: 03/08/2017 Aprobado: 22/11/2017 En línea: 07/12/2017
}

Citar como: Accinelli RA, Herrera S, Canales Y, López LM. Malabsorción de lactosa en adolescentes de un club de fútbol. Rev Peru Med Exp Salud Publica. 2017;34(4): 755-6.doi: 10.17843/rpmesp.2017.344.3037 de $55,1 \%$. Otros autores peruanos muestran que la MAL se presenta en más del $50 \%$ de personas estudiadas (Tabla 1).

En América Latina las prevalencias no distan mucho de este resultado, países como Chile, Colombia y Ecuador presentan MAL en $51 \%, 59 \%$ y $65 \%$ respectivamente, mientras que en Bolivia la prevalencia es mayor (85\%). En el sur de Europa (70-80\%), entre nativos americanos $(80-95 \%)$ y en el sur de Asia (90\%) se presentan las prevalencias más elevadas, mientras que en el norte de Europa se encuentra una menor prevalencia (menor de 5\%). Estas diferencias a nivel mundial pueden ser explicadas en función de la carga genética, las prácticas culturales y los mecanismos evolutivos a los que han estado expuestos.

El momento del inicio de consumo de leche en la dieta de la población adulta también parece explicar estas diferencias. En Europa se inició aproximadamente hace 7500 años y por selección positiva apareció la persistencia de la lactasa hace 5000 años. En cambio, el consumo de productos lácteos en la edad adulta en América Latina ocurrió hace menos de 500 años cuando llegaron los europeos.

Existen diferentes métodos para diagnosticar malabsorción a lactosa. Las pruebas de actividad de lactasa en biopsias de mucosa del duodeno se consideran como el estándar de referencia para la deficiencia de lactasa primaria, que es la más frecuente ${ }^{(1)}$. El test de tolerancia a lactosa tiene sensibilidad y especificidad bastante variable, lo cual resulta en una de las principales limitantes de este estudio.

No existen recomendaciones globales para el consumo de leche de vaca, pero muchos países han desarrollado directrices considerando la disponibilidad local de alimentos, el costo, el estado nutricional, los patrones de consumo y los hábitos alimenticios. En Chile se recomienda 3 a 4 vasos de leche al día en niños y adolescentes, y 2 a 3 en adultos. En países de Europa de 3 a 5 vasos en adultos y de 500 a $1000 \mathrm{~mL}$ en niños y adolescentes, y en Asia entre $300 \mathrm{~mL}$ y $750 \mathrm{~mL}$ para niños y adultos ${ }^{(5)}$.

Las personas con MAL pueden ingerir hasta $12 \mathrm{~g}$ de lactosa sin desarrollar síntomas, debido a la adaptación del colon a la ingestión regular de lactosa. Dosis mayores a 18 $\mathrm{g}$ parecen ser bien toleradas si se consume la leche con otros nutrientes, mientras que en la mayoría de personas cantidades mayores de $50 \mathrm{~g}$ producen síntomas. Considerar

Tabla 1. Frecuencia de malabsorción de lactosa según diversos autores en Perú

\begin{tabular}{lcc}
\hline Autores & Edad en años & $\%$ \\
\hline Calderón C. & 21 a 29 & 68 \\
León-Barúa R. et al. & - & 63,3 \\
Figueroa R. et al. & 16 a 54 & 66 \\
Reyes C. et al. & 18 a 29 & 65,1 \\
Namizato C. et al. & 4 a 18 & 65,7 \\
\hline
\end{tabular}


solo restringir lactosa funciona en casos que se presente únicamente intolerancia a la lactosa, pero no en aquellos con alguna otra patología gastrointestinal asociada. Otra alternativa es la del reemplazo de la enzima lactasa. Esta se obtiene del Aspergillus oryzae o de Kluyveromyces lactis. Así, al descomponer la lactosa en sus respectivos monosacáridos se logra su absorción eficiente ${ }^{(1)}$. También se ha planteado el uso de probióticos que alteren la flora intestinal, pero aún se requiere de más estudios.

En conclusión, se encontró una alta frecuencia de MAL entre jugadores de fútbol adolescentes similar a resultados encontrados en población general.

Contribución de los autores: RAA y LML han participado en la concepción y diseño del artículo, interpretación de datos, redacción del artículo y aprobación de su versión final. SH y YC han participado en el análisis e interpretación de datos, redacción del artículo y aprobación de su versión final.

Fuentes de financiamiento: este estudio fue financiado por Pesquera Capricornio S.A.

Conflictos de interés: los autores declaran no tener conflictos de interés en la publicación de este artículo.

\section{REFERENCIAS BIBLIOGRÁFICAS}

1. Deng Y, Misselwitz B, Dai N, Fox M. Lactose Intolerance in Adults: Biological Mechanism and Dietary Management. Nutrients. 2015; 7(9):8020-35.

2. Storhaug CL, Fosse SK, Fadnes LT. Country, regional, and global estimates for lactose

3. malabsorption in adults: a systematic review and meta-analysis. Lancet Gastroenterol Hepatol. 2017; 2(10):738-46.

4. Domínguez-Jiménez JL, Fernández-Suárez A. Diagnosis of lactose intolerance. Med Clin (Barc). 2017; 148(6):262-4.

5. Domínguez-Jiménez JL, Fernández-Suárez A, Ruiz-Tajuelos S, PuenteGutiérrez JJ, Cerezo-Ruiz A. Lactose tolerance test shortened to 30 minutes: An exploratory study of its feasibility and impact. Rev Esp Enferm Dig. 2014; 106(6):381-5.

6. Food and Agriculture Organization of The United Nations. Milk and dairy products in human nutrition. Rome: FAO; 2013.

Correspondencia: Roberto Accinelli Tanaka.

Dirección: Av. Honorio Delgado 430, Urb. Ingeniería, Lima

15102, Perú.

Teléfono: (51) 998119480

Correoelectrónico: roberto.accinellit@upch.pe

\section{jAhora puede subir su artículo, a través de nuestro sistema Open Journal System (OJS)!}

www.rpmesp.ins.gob.pe 